Proceedings of the 37th Polish Seminar on Positron Annihilation, Lạdek-Zdrój 2007

\title{
Production of Cold Positronium Atoms
}

\author{
R.S. Brusa ${ }^{a}$, G. Consolati ${ }^{b, *}$, A. Dupasquier ${ }^{b}$, \\ M.G. GiammarChi ${ }^{c}$, S. MARIAZzi ${ }^{a}$ AND F. QUASSO ${ }^{b}$ \\ ${ }^{a}$ Dipartimento di Fisica, Universitá di Trento \\ Via Sommarive 14, 38100 Trento, Italy \\ ${ }^{b}$ Dipartimento di Fisica, Politecnico di Milano \\ Piazza Leonardo da Vinci, 32, 20133 Milano, Italy \\ ${ }^{c}$ Infn Milano, Via Celoria 16, 20133 Milano, Italy
}

Positronium will play a primary role in the next generation of antimatter experiments through the following antihydrogen production reaction: $\overline{\mathrm{p}}+\mathrm{Ps}^{*} \rightarrow \overline{\mathrm{p}} \mathrm{e}^{+}+\mathrm{e}^{-}$. In order to study antimatter physical properties (CPT (charge, parity, time) invariance and principle of equivalence test) it is necessary to keep this system at the lowest possible (sub-kelvin) temperatures. This requires the generation of a suitable flux of cold Ps atoms in a vacuum, a non-trivial requirement at the light of the present experimental results. In this paper we discuss the state of the actual knowledge on positronium formation and consequent emission from metallic surfaces and insulators and we show the opportunity to use suitable porous materials to cool positronium through collisions with the inner walls of the pores. We get a rough indication on the geometrical parameters of the pore and we propose a simple experiment to obtain the kinetic energy - and therefore the equivalent temperature - of emitted positronium without using a positron beam.

PACS numbers: 78.70.Bj, 36.10.Dr, 36.10.Gv

\section{Introduction}

Although positronium (Ps) is mainly known as a probe in material science studies [1-3], since its discovery it has been used for fundamental research, as in e.g. quantum electrodynamics (QED) precision tests [4]. In this connection, Ps was proposed [5] as an intermediate step in the production of antihydrogen through the following charge exchange reaction:

$$
\overline{\mathrm{p}}+\mathrm{Ps}^{*} \rightarrow\left(\overline{\mathrm{p}} \mathrm{e}^{+}\right)^{*}+\mathrm{e}^{-} \text {. }
$$

Antihydrogen - the first antimatter atom whose low energy (10-100 K) synthesis was successfully obtained for the first time in $2002[6,7]$ by following a different approach with respect to (1) - is considered an important system to carry out a test on CPT invariance theorem as well as for gravitation (weak equivalence principle) checks. To this purposes, the AEGIS collaboration aims to generate cold antiatoms $(\approx 100 \mathrm{mK})[8]$ using $(1)$. The components of the reaction in particular, Ps - should be at the lowest temperature compatible with their production. Furthermore, since the cross section of the reaction increases roughly

*corresponding author; e-mail: giovanni.consolati@polimi.it 
as $n^{4}$ - where $n$ is the Ps principal quantum number - it is convenient to have Ps in a highly excited state, with $20 \leq n \leq 40$. This process could be obtained by means of a two-pulses laser excitation suitably synchronised with Ps emission from a target, able to bring Ps in a Rydberg state. In the present work we will discuss a few problems concerning the first stage of the process which should generate antihydrogen, that is, the production of a gas of cold Ps atoms.

\section{Ps energy requirements}

Positrons implanted in a solid can be emitted in vacuum as positronium after electron capture. The amount of Ps formed depends on the nature of the material, on the implantation depth and on the temperature of the target; in favourable conditions and in the high temperature limit, it may approach $100 \%$ [9]. A sufficient yield of cold Ps should be emitted from a converter, in order to limit the Ps kinetic energy associated to the centre-of-mass motion $E_{\mathrm{CM}}$, for three reasons. Indeed, first of all a sufficient value of the $\bar{p} / \mathrm{Ps}$ charge exchange crosssection is obtained when the relative velocity is not higher than the orbital velocity of the $\mathrm{e}^{+}$in the Rydberg Ps atom, which means $E_{\mathrm{CM}} \approx \frac{4 \times 6.8 \mathrm{eV}}{n^{2}}$. By choosing $n$ in the interval from 20 to 40 , the corresponding interval for $E_{\mathrm{CM}}$ is from $68 \mathrm{meV}$ to $16 \mathrm{meV}$. This last value - corresponding to a Ps equivalent temperature of the order of $200 \mathrm{~K}$ - could be realistically obtained.

In a second place, the density of initial Ps gas cloud should fit the power of the laser used for exciting the atoms. Also in the most unfavourable case of Ps with $E_{\mathrm{CM}}=68 \mathrm{meV}$, the spread of the emitted Ps gas cloud is as short as $2 \mathrm{~mm}$ if Ps is formed by $\mathrm{e}^{+}$pulses with a bunching time of $20 \mathrm{~ns}$. Assuming this spread, a value around $10^{15} \mathrm{Ps} / \mathrm{m}^{3}$ can be obtained with the formation of $10^{7} \mathrm{Ps}$ atoms per $\mathrm{e}^{+}$pulse. Finally, the Ps equivalent temperature should be as low as possible, in order to minimize a transfer of a fraction $m_{\mathrm{e}} / m_{\overline{\mathrm{p}}}$ of $E_{\mathrm{CM}}$ to antihydrogen atoms just formed.

We point out that the converter must operate at the lowest possible temperature, in order to avoid to heat the near antiproton cloud.

\section{Positron to Ps conversion}

In metals and in semiconductors Ps formation is mainly a surface process: a positron that reaches the surface can capture an electron from the solid being emitted as a Ps atom. Energy constraints require that

$$
E_{\mathrm{B}}+E_{\mathrm{k}}>\Phi_{-}+\Phi_{+},
$$

where $E_{\mathrm{B}}=6.8 \mathrm{eV}$ is the Ps binding energy; $E_{\mathrm{k}}$ is the kinetic energy of the positron after returning to the surface; $\Phi_{-}, \Phi_{+}$are the work functions of the electron and of the positron, respectively.

The difference $\Phi_{-}+\Phi_{+}-E_{\mathrm{B}}$ is the Ps formation potential $W$. When $W<0$, adiabatic Ps emission occurs at any temperature. When also $\Phi_{+}<0$, direct positron emission is a process in competition with adiabatic Ps emission. Ag, Au and $\mathrm{Pb}$ are known cases for which Ps emission does not compete with $\mathrm{e}^{+}$emission $\left(W<0, \Phi_{+}>0\right)[10]$. 
When Ps is produced by positrons trapped at the surface by image charge forces, thermally activated emission occurs in addition to adiabatic emission. Concerning Ag, adiabatic emission (low temperature limit) gives a $\mathrm{Ps} / \mathrm{e}^{+}$conversion yield of about $30 \%$; the thermal contribution, in the high temperature limit, may provide the difference to $100 \%$ [11]. Ps yield decreases with the temperature of the target; anyway, its equivalent temperature may be much higher than that of the converter.

For insulators, surface Ps formation is expected from epithermal positrons, since thermalisation of positrons is less efficient than in a metal: a larger flux of positrons return to the surface with sufficient kinetic energy to form Ps. This process is known to occur in $\mathrm{Al}_{2} \mathrm{O}_{3}$ (conversion yield 28\%) and $\mathrm{MgO}$ (conversion yield 24\%) [12].

Bulk Ps formation also occurs in several insulators. In general, $E_{\mathrm{B}}^{\text {solid }}<$ $E_{\mathrm{B}}^{\text {vacuum }}=6.8 \mathrm{eV}$. The bound $\mathrm{e}^{+}-\mathrm{e}^{-}$pair is a mobile system, stable against dissociation provided that the centre-of-mass energy is smaller than the binding energy. Bulk Ps formation may occur during the $\mathrm{e}^{+}$slowing down, that is, through the Ore gap. Additional bulk formation of Ps is given by thermalised positrons that encounter spur electrons. A Ps atom will eventually reach the surface with a residual kinetic energy $E_{\mathrm{CM}}^{\text {solid }}$ that depends on the depth of formation, if not annihilated during the migration process by a defect or self-trapped in a phonon cloud. Bulk formation of Ps is known to be the only important channel for Ps emission in ice [13] and ionic crystals [14] (conversion yields 30-40\%) and to be the dominant one in $\mathrm{a}_{-} \mathrm{SiO}_{2}$ (conversion yield $72 \%$, additional to another $12 \%$ coming from surface formation) [12]. Surface or bulk formation depend on the temperature of the sample only indirectly, through temperature effects on migration and trapping. Ps conversion yield could be expected to stay high even at cryogenic temperatures, but, again, Ps equivalent temperature is not necessarily related to the temperature of the target.

Therefore, present knowledge supports the following remarks:

- A high Ps yield, as possibly obtained with some metals and elemental semiconductors, is lost when the target is operated at room temperature or below. Insulators produce best yields at low temperatures. Nevertheless, if the highest yield is not essential, metals should not be discarded in advance as possible targets: contrarily to insulators they do not accumulate charges and this does not generate electric fields which disturb the region where antihydrogen must be formed.

- Efficient $\mathrm{e}^{+} /$Ps conversion cannot be coupled with emission of Ps with kinetic energy of a few tens of meV. Rather, the desired low energy of Ps can be obtained by cooling after the emission from the surface of the converter.

\section{Ps cooling}

Ps energy loss by elastic collisions with gas molecules and condensed insulating matter can be used for cooling the hot Ps atoms emitted by a converter 
surface in form of a bidimensional array of tubular pores with an open end. Ps, emitted inside the pore with an energy of the order of $1 \mathrm{eV}$, would thus emerge in the vacuum from the open end of the pore at the desired energy after a sufficient number of collisions against the walls of the pore. Partial loss by annihilation cannot be avoided, but it may not be dramatic. The cooling process should occur only by elastic collisions with the atoms at the surface of the solid. The duration $\Delta t$ of a collision is expected to be of the order of the ratio between the thickness of the first atomic layer and the Ps velocity $\left(4 \times 10^{4} \mathrm{~m} / \mathrm{s}\right.$ at $\left.10 \mathrm{meV}\right)$; thus $\Delta t \approx 5 \times 10^{-15} \mathrm{~s}$, shorter than the inverse of typical Debye frequencies. During such a time the energy transferred from bouncing Ps does not spread from the atomic cluster which undergoes the collision and these atoms can be considered as free. Therefore, phonon effects on the computation of the energy transfer can be neglected. Within this framework the maximum fractional energy transfer in a single collision of a Ps atom (mass $2 m_{\mathrm{e}}$ ) with an object of effective mass $M_{\mathrm{eff}} \gg m_{\mathrm{e}}$ is given by

$$
\frac{\Delta E}{E} \approx \frac{8}{2+M_{\mathrm{eff}} / m_{\mathrm{e}}} \approx \frac{8 m_{\mathrm{e}}}{M_{\mathrm{eff}}} .
$$

The effective mass $M_{\text {eff }}$ can be approximated as $M_{\text {eff }}=p M$, where $p$ is the number of surface atoms exposed to the impact. $p$ can be calculated in terms of the ratio between the de Broglie wavelength $\lambda$ of the Ps atom and the interatomic distance $a$ :

$$
p \approx\left(\frac{\lambda}{a}\right)^{2}=\left(\frac{\eta}{a \sqrt{4 m E}}\right)^{2}=\frac{1}{16 \pi^{2}} \frac{m c^{2}}{E}\left(\frac{\lambda_{\text {Compton }}}{a}\right)^{2} .
$$

In the high energy limit $\lambda<a$ and $p=1$. Nagashima et al. [15] estimate values of the effective mass between 20 to $40 \mathrm{amu}$, according to the Ps energy.

Cooling a Ps atom from $1 \mathrm{eV}$ to $10 \mathrm{meV}$ requires about $10^{5}$ collisions. A higher number of collisions would imply excessive Ps loss by pickoff annihilation against the pore surface. Indeed, the probability of pick-off annihilation in a single collision, as estimated from the known values of the pick-off rates of Ps atoms trapped in voids, is near to $10^{-5}$. With $10^{5}$ collisions, the pick-off loss is thus expected to be around 60 to $70 \%$. Moreover, $25 \%$ of the Ps formed is in the para state which promptly disappears by self-annihilation. Only a fraction $F=1-\exp (-\lambda t)$ of the remaining ortho-Ps will survive at the exit of the pore, where $t$ is the average travel time, which depends on the geometry of the pore and $\lambda=\frac{1}{\tau_{\mathrm{oPs}}}+\lambda_{\text {po }}$, by taking into account both pick-off and self-annihilation $\left(\tau_{\mathrm{oPs}}=142 \mathrm{~ns}\right)$. Therefore, the pore must be suitably chosen to keep $t \ll 1 / \lambda$. Length $L$ and diameter $d$ of the pore (framed as a cylinder) can be correlated to the number $k$ of collisions: a stochastic approximation for the random walk of a Ps colliding against the walls of the pore and diffusing toward the open end supplies the relation $L=d \sqrt{k}$. Within the same approximation the travel time $t$ is related to the pore diameter $d$ and to the average Ps speed $v: t=k d \sqrt{2} / v$. Travel time and average Ps speed can be assumed $t \approx \tau_{\mathrm{oPs}} / 10, v=4 \times 10^{4} \mathrm{~m} / \mathrm{s}$. The number 
of collisions strongly depends on the effective mass of atoms on pore walls: we can estimate for $k$ a range $k=1 \times 10^{5}-3 \times 10^{5}$ [16]. With the above data we obtain $d \approx 4-12 \mathrm{~nm}$ and $L \approx 2-4 \mu \mathrm{m}$. Obviously, these numbers should be considered only as a first orientation on the choice of the possible converters among structures that are commercially available or that can be produced with a reasonable effort.

A metallic converter would be more efficient from the point of view of cooling, since a single collision of a Ps atom with a free electron at the surface of the metal produces a fractional energy loss of $50 \%$, thereby reducing the number of collisions to less than 100. However, the lack of experimental data on cooling by collisions with metallic surfaces suggests caution. For a Ps entering into a dense free electron gas the preferential binding of the positron with an individual electron is lost, due to fast rearrangement of the electron cloud; the positron will then be annihilated, trapped at the surface by image forces or reemitted as a bare positron. Therefore, the probability that the impinging ortho-Ps is re-emitted as ortho-Ps is expected to be well below unity, with a strong reduction of the Ps flux.

\section{Preliminary experimentation}

Facilities with a DC slow positron beam are available in Italy, both at the Milano Politecnico and at the Trento University; unfortunately, neither of them is actually equipped for carrying out time-of-flight experiments. This makes impossible to know the Ps energy spectrum, which is a drawback in the preliminary phases of the experiment devoted to select the best converter. We aim to achieve time resolution through an upgrading of the present equipment with a time spectrometer using as a trigger the secondary electrons ejected by each impinging positron.

Meanwhile, an attempt to perform time-of-flight measurements without a positron beam is currently planned by us. The positron source is mounted in the usual sandwich geometry between two discs of a target (a few micron thick) inside a small vacuum chamber. While most of the positrons will travel through the target, a small fraction of them will form Ps in the pores. Ps atoms coming out of the pores in the vacuum chamber will either annihilate in flight or hit a pair of metallic diaphragms symmetrically mounted at variable distance $x$ from the source-target sandwich.

Two experiments can be performed with such a simple apparatus:

1) Measurement of the time annihilation spectrum over a time range extended beyond the mean life of ortho-Ps in a vacuum. The time spectra should contain: (a) a very large distribution coming from all the positrons that do not form Ps and from Ps formed and annihilated inside the pores. These components would extend in the time region between 0 and a few ns; (b) an exponentially decreasing component corresponding to ortho-Ps annihilation in flight, with very long lifetime; (c) a delayed distribution corresponding to ortho-Ps atoms which annihilate when colliding with the diaphragms after a time-of-flight $t_{\mathrm{f}}=x / v$. The measurement of $t_{\mathrm{f}}$ would give the value of $v$ and therefore information on the Ps kinetic energy. 
2) Measurement of the number of $3 \gamma$ annihilations by means of a triple coincidence setup. The number of triple coincidence events should grow toward an asymptote when the distance $x$ increases from 0 to a few $\mathrm{mm}$, since the travel distance of a Ps atom of $10 \mathrm{meV}$ in vacuum is smaller than $6 \mathrm{~mm}$. The slope of the curve contains information on the velocity spectrum of Ps.

Of course, experimental difficulties can be expected, mainly due to the low probability of Ps formation with consequent faint intensity of the corresponding component, or the low efficiency of the three quantum yield technique. In spite of these drawbacks, the experiment is appealing owing to its simplicity.

\section{References}

[1] Principles and Applications of Positron and Positronium Chemistry, Eds. Y.C. Jean, P.E. Mallon, D.M. Schrader, World Scientific, New Jersey 2003.

[2] R. Krause-Rehberg, H. Leipner, Positron Annihilation in Semiconductors, Springer Series in Solid-State Sciences, Vol. 127, Springer Verlag, Berlin 1999.

[3] Positron Spectroscopy of Solids, Eds. A. Dupasquier, A.P. Mills, Jr , North Holland, Amsterdam 1995.

[4] R.S. Conti et al., in: Very High Resolution Photoelectron Spectroscopy, Springer Lect. Not. in Physics, Vol. 570, Ed. S. Huefner, Springer, Heidelberg 2001, p. 103.

[5] M. Charlton, J.W. Humberston, Positron Physics, Cambridge Monographs on Atomic, Molecular and Chemical Physics, vol. 11, Cambridge University Press, Cambridge 2000, p. 372.

[6] M. Amoretti, C. Amsler, G. Bonomi, A. Bouchta, P. Bowe, C. Carraro, C.L. Cesar, M. Charlton, M.J.T. Collier, M. Doser, V. Filippini, K.S. Fine, A. Fontana, M.C. Fujiwara, R. Funakoshi, P. Genova, J.S. Hangst, R.S. Hayano, M.H. Holzscheiter, L.V. Jorgensen, V. Lagomarsino, R. Landua, D. Lindelf, E. Lodi Rizzini, M. Macri, N. Madsen, G. Manuzio, M. Marchesotti, P. Montagna, H. Pruys, C. Regenfus, P. Riedler, J. Rochet, A. Rotondi, G. Rouleau, G. Testera, A. Variola, T.L. Watson, D.P. van der Werf, Nature 419, 456 (2002).

[7] G. Gabrielse, N.S. Bowden, P. Oxley, A. Speck, C.H. Storry, J.N. Tan, M. Wessels, D. Grzonka, W. Oelert, G. Schepers, T. Sefzick, J. Walz, H. Pittner, T. W. Hnsch, E.A. Hessels, Phys. Rev. Lett. 89, 213401 (2002).

[8] A. Kellerbauer et al., Nucl. Instr. Methods. Phys. Res. B 266, 351 (2008).

[9] A.P. Mills, Jr., Phys. Rev. Lett. 41, 1828 (1978).

[10] M.J. Puska, R.M. Nieminen, Rev. Mod. Phys. 33, 841 (1994).

[11] K.G. Lynn, D.O. Welch, Phys. Rev. B 22, 99 (1980).

[12] S. Van Petegem, C. Dauwe, T. Van Hoecke, J. De Baerdemaeker, D. Segers, Phys. Rev. B 70, 115410 (2004).

[13] M. Eldrup, A. Vehanen, P.J. Shultz, K.G. Lynn, Phys. Rev. B 32, 7048 (1985).

[14] M. Toumisaari, R.H. Howell, T. McMullen, Phys. Rev. B 40, 2060 (1989).

[15] Y. Nagashima, Y. Morinaka, T. Kurihara, Y. Nagai, T. Hyodo, T. Shidara, K. Nakahara, Phys. Rev. B 58, 12676 (1998).

[16] C. He, T. Ohdaira, N. Oshima, M. Muramatsu, A. Kinomura, R. Suzuki, T. Oka, Y. Kobayashi, Phys. Rev. B 75, 195404 (2007). 